\title{
Terahertz-wave generation from surface phonons at forbidden frequencies of lithium niobate
}

\author{
Jun-ichi Shikata $^{1, \text { a) }}$, Seigo Ohno ${ }^{2,3}$, and Hiroaki Minamide ${ }^{3}$
}

Abstract This paper describes terahertz (THz)-wave generation within forbidden bands in polar crystals, focusing on the $A_{1}$ phonon modes in lithium niobate. This material exhibits two negative-permittivity frequency ranges at 7.4-12.7 THz and $18.8-25.6 \mathrm{THz}$ for the lowest and highest $\mathrm{A}_{1}$ modes, respectively. Exploiting the finite-difference time-domain simulations, we demonstrate that both the surface phonon modes can be radiative with a structured grating. Fourier analyses of the radiative fields reveal the relevant peaks in the spectrum as well as the dispersion relations. Our results provide a novel method for coherent THz-wave sources at unexplored $\mathrm{THz}$ frequencies.

Keywords: terahertz-wave generation, surface phonon, lithium niobate, negative permittivity, $\mathrm{A}_{1}$ mode

Classification: Electromagnetic theory

\section{Introduction}

Recently, a variety of compact coherent terahertz (THz)wave sources have been developed based on photonics and ultrafast electronics. These sources include THz-wave generators using nonlinear optics $[1,2,3,4,5,6,7,8,9]$, photoconductive antennas $[10,11,12], \mathrm{THz}$ quantum cascade lasers [13, 14, 15], TUNNETT diodes [16, 17], unitraveling-carrier photodiodes $[18,19,20]$, resonant tunneling diodes [21, 22, 23], and plasmonic THz emitters [24, 25]. However, the interaction of optical phonons with photons frequently restricts the $\mathrm{THz}$ waves available, because it results in forbidden bands [26], where the $\mathrm{THz}$ waves are unable to propagate over a long distance in bulk media. In $\mathrm{THz}$-wave generation by stimulated polariton scattering in $\mathrm{LiNbO}_{3}$ (LN) $[1,2,3,4,5,6]$, the tuning range is limited to $0.7-3.0 \mathrm{THz}$ due to the substantial absorption loss of the $\mathrm{THz}$ waves interacting with the lowest $\mathrm{A}_{1}$ phonon mode (7.4 $\mathrm{THz}$ ).

A possible way to generate $\mathrm{THz}$ waves at forbidden frequencies in bulk is to couple out the excited surface phonons [27]. In fact, thermally excited surface phonons on $\mathrm{SiC}$ gratings have been shown to exhibit infrared emission [28], and the spectra of this emission cover a wide

\footnotetext{
${ }^{1}$ College of Engineering, Nihon University, Koriyama 9638642, Japan

${ }^{2}$ Graduate School of Science, Tohoku University, Aoba-ku, Sendai 980-8578, Japan

${ }^{3}$ RIKEN Center for Advanced Photonics (RAP), RIKEN, Aobaku, Sendai 980-0845, Japan

a) shikata.junnichi@nihon-u.ac.jp
}

DOI: $10.1587 /$ elex.17.20200133

Received April 6, 2020

Accepted April 16, 2020

Publicized April 30, 2020

Copyedited June 10, 2020 frequency range (more than several $\mathrm{THz}$ ) in the forbidden band. Since surface waves have long-range coherence [29], coherent emissions occur even with incoherent excitations.

In this paper, we explore $\mathrm{THz}$-wave generation from surface phonons, focusing on the $\mathrm{A}_{1}$ modes in $\mathrm{LN}$ crystals. We discover surface phonon modes arising from the lowest and highest $\mathrm{A}_{1}$ modes. Finite-difference time-domain simulations reveal the $\mathrm{THz}$-wave radiation from a grating surface. The emission spectra as well as the dispersion relations are found using Fourier analyses.

\section{Theoretical background}

In the bulk of a polar crystal, the interaction between transverse optical (TO) phonons and transverse photons results in coupled modes, called polaritons [26]. With respect to a simple single-oscillator (two-atom) model in lossless media, the polariton wavevector (i.e., dispersion relation) $k(\omega)=(\omega / c) \sqrt{\varepsilon(\omega)}$ is determined using the following dielectric function:

$$
\varepsilon(\omega)=\varepsilon_{\infty}+\frac{S \omega_{T}^{2}}{\omega_{T}^{2}-\omega^{2}}=\frac{\varepsilon_{\infty}\left(\omega_{L}^{2}-\omega^{2}\right)}{\omega_{T}^{2}-\omega^{2}}
$$

where $\varepsilon_{\infty}$ is the permittivity for high frequencies, $S$ is the oscillator strength, $\omega_{T}$ is the frequency of the TO phonon, and $\omega_{L}$ is the frequency of the longitudinal optical phonon. As $\varepsilon(\omega)<0$ at $\omega_{T}<\omega<\omega_{L}$, the wavevector is purely imaginary $(k=i|k|)$; thus, the amplitude of the electromagnetic wave decays as $\exp (-|k| x)$ according to its distance from the surface (forbidden band). The zero point of Eq. (1) $\left[\varepsilon(0)=\varepsilon_{0}\right]$ provides the well-known Lyddane-Sachs-Teller (LST) relation as follows:

$$
\frac{\omega_{L}}{\omega_{T}}=\sqrt{\frac{\varepsilon_{0}}{\varepsilon_{\infty}}}
$$

Although the electromagnetic waves at the forbidden frequencies cannot propagate for long in bulk, surface waves (known as surface phonons [27]) can freely propagate along the surface with transverse magnetic (TM) polarization. On a flat surface between the crystal and air, the electromagnetic boundary conditions yield the following surface phonon wavevector:

$$
k_{S P}(\omega)=\frac{\omega}{c} \sqrt{\frac{\varepsilon(\omega)}{\varepsilon(\omega)+1}}
$$

The dispersion curve of the surface phonon emerges from $\omega_{T}[\varepsilon(\omega)=-\infty]$, whereas the upper limit $\omega_{s}$ is specified by 


$$
\omega_{s}=\omega_{T} \sqrt{\frac{\varepsilon_{0}+1}{\varepsilon_{\infty}+1}}
$$

from the condition $k_{S P}=\infty[\varepsilon(\omega)=-1]$ in Eq. (3).

It is worth noting that the dispersion curve is below the light line $k=\omega / c$ because $\varepsilon(\omega) /(\varepsilon(\omega)+1)>1$ in Eq. (3) at $-\infty<\varepsilon(\omega)<-1$. Since the energy and momentum conservations do not hold simultaneously, THz-wave emissions do not occur from surface phonons on a flat surface. However, if a Bragg grating (Fig. 1) is structured on the surface, the radiative coupling becomes possible in accordance with the condition of momentum conservation:

$$
k_{S P}(\omega)=\frac{\omega}{c} \sin \theta+m \frac{2 \pi}{\Lambda}
$$

where $\theta$ is the radiation angle, $m$ is the diffraction order (an integer), and $\Lambda$ is the grating period.

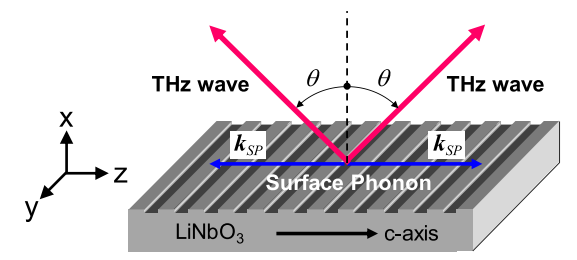

Fig. 1 Schematic diagram of THz-wave generation from surface phonons on a grating surface of $\mathrm{LN}$.

\section{Dispersion relations of $A_{1}$ modes in $\mathbf{L N}$}

\subsection{Bulk polariton modes}

Since an $\mathrm{LN}$ crystal has four $\mathrm{A}_{1}$ modes, the nth mode can be designated as $A_{1}{ }^{(n)}$ in Table I. We used the multioscillator model of the dielectric function, including the non-negligible damping terms, described as

$$
\varepsilon(\omega)=\varepsilon_{\infty}+\sum_{n} \frac{S_{n} \omega_{T_{n}}^{2}}{\omega_{T_{n}}^{2}-\omega^{2}-i \Gamma_{n} \omega}
$$

where $\Gamma_{n}$ is the damping coefficient.

Table I Optical parameters of $\mathrm{A}_{1}$ modes in LN [30].

\begin{tabular}{c|c|c|c|c||c}
\hline mode & $\omega_{T}\left(\mathrm{~cm}^{-1}\right)$ & $S$ & $\Gamma\left(\mathrm{cm}^{-1}\right)$ & $\varepsilon_{\infty}$ & $\omega_{L}\left(\mathrm{~cm}^{-1}\right)$ \\
\hline $\mathrm{A}_{1}{ }^{(1)}$ & 248 & 16.0 & 21 & & 273 \\
\hline $\mathrm{A}_{1}{ }^{(2)}$ & 274 & 1.0 & 14 & \multirow{4}{*}{4.6} & 306 \\
\hline $\mathrm{A}_{1}{ }^{(3)}$ & 307 & 0.16 & 25 & & 423 \\
\hline $\mathrm{A}_{1}{ }^{\left({ }^{(4)}\right.}$ & 628 & 2.55 & 34 & & 869 \\
\cline { 1 - 3 } \cline { 5 - 6 }
\end{tabular}

Fig. 2 shows the dielectric properties calculated from Eq. (6). The strong resonances $\left(\mathrm{A}_{1}{ }^{(1)}, \mathrm{A}_{1}{ }^{(4)}\right)$ almost determine the dispersive and loss characteristics of the polaritons in Figs. 2(a) and 2(b), whereas the weak resonances $\left(\mathrm{A}_{1}{ }^{(2)}\right.$, $\left.\mathrm{A}_{1}{ }^{(3)}\right)$ contribute less. The resulting negative-permittivity regions emerge at 7.4-12.7 $\mathrm{THz}$ and $18.8-25.6 \mathrm{THz}$ [the upper part of Fig. 2(a)]. Accordingly, we focused on the lowest and highest $A_{1}$ modes to study the surface phonon bands and their emissions, as shown below.

\subsection{Surface phonon modes on a flat surface}

To solve Eq. (3) for the lossy case [Eq. (6)], we employed the complex frequency scheme [31], where the frequency
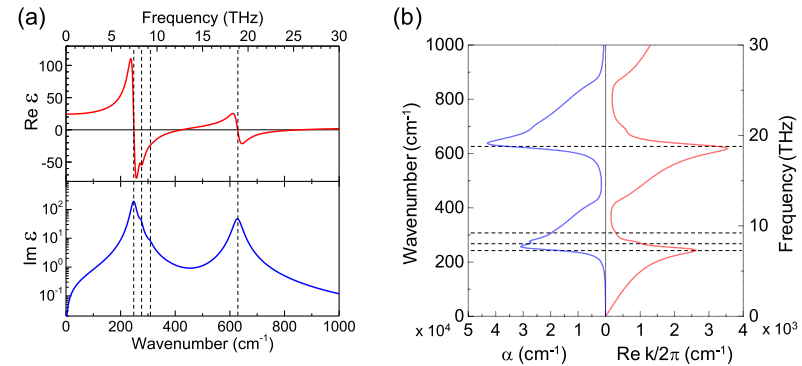

Fig. 2 Dielectric properties associated with $A_{1}$ modes in bulk LN. (a) Complex dielectric functions. (b) Dispersion relations of the polaritons. The frequency is assumed to be real. The dashed lines denote the TO phonon frequencies $\left(\omega_{T}\right)$. The absorption coefficient is defined by $\alpha=2$ $\operatorname{Im} k$.

was assumed to be complex $\left(\omega=\omega^{\prime}-i \omega^{\prime \prime}\right.$, such that the amplitude decay was expressed as $\exp \left(-\omega^{\prime \prime} t\right)$. We modified Eq. (3) to the following system of nonlinear equations:

$$
\begin{aligned}
k_{S P} & =\operatorname{Re}\left[\frac{\omega^{\prime}-i \omega^{\prime \prime}}{c} \sqrt{\frac{\varepsilon\left(\omega^{\prime}-i \omega^{\prime \prime}\right)}{\varepsilon\left(\omega^{\prime}-i \omega^{\prime \prime}\right)+1}}\right] \\
0 & =\operatorname{Im}\left[\frac{\omega^{\prime}-i \omega^{\prime \prime}}{c} \sqrt{\frac{\varepsilon\left(\omega^{\prime}-i \omega^{\prime \prime}\right)}{\varepsilon\left(\omega^{\prime}-i \omega^{\prime \prime}\right)+1}}\right]
\end{aligned}
$$

Eq. (7) as well as Eqs. (6) and (8) were numerically solved for each fixed real wavevector $k_{S P}$.

Fig. 3 illustrates the resulting dispersion relations of the $A_{1}$ surface phonon modes. In Fig. 3(a), the lower branch emerges from $\omega_{T}=7.4 \mathrm{THz}$, asymptotically approaching the upper limit $\omega_{s}=14.6 \mathrm{THz}$. Moreover, in Fig. 3(b), the lower branch extends from $\omega_{T}=18.8 \mathrm{THz}$ to $\omega_{s}=22.7$ THz. Both the upper limits $\left(\omega_{s}\right)$ agree well with those resulting from Eq. (4). In this way, we found that both the lower branches in Figs. 3(a) and 3(b) were of surface phonons that were relevant to the $A_{1}{ }^{(1)}$ and $A_{1}{ }^{(4)}$ modes, respectively.
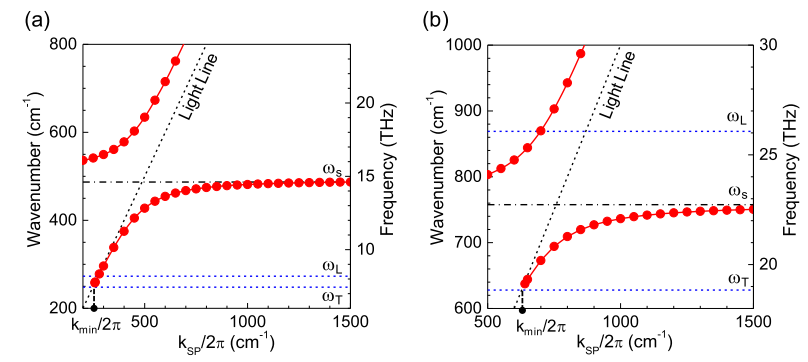

Fig. 3 Dispersion relations of surface phonon modes on a flat surface. (a) $\mathrm{A}_{1}{ }^{(1)}$ mode, $\omega_{s}=14.6 \mathrm{THz}$. (b) $\mathrm{A}_{1}{ }^{(4)}$ mode, $\omega_{s}=22.7 \mathrm{THz}$. The surface wavevector $k_{S P}$ is assumed to be real.

Note that the $\mathrm{A}_{1}{ }^{(4)}$ surface phonon band (18.8-22.7 THz) is within the negative-permittivity region $(18.8-25.6 \mathrm{THz})$ [Fig. 2(a)]; however, $\omega_{s}=14.6 \mathrm{THz}$ of the $\mathrm{A}_{1}{ }^{(1)}$ mode exceeds the upper boundary of the forbidden band (12.7 $\mathrm{THz}$ ). This anomaly results from the large oscillator strength $S$ (i.e., $\varepsilon_{0}$ ) of the $\mathrm{A}_{1}{ }^{(1)}$ mode, which results in the breakdown of the LST relation [32]. Indeed, for the $A_{1}{ }^{(1)}$ mode, the LST relation (2) is broken $\left(\omega_{L} / \omega_{T}=1.1, \sqrt{\varepsilon_{0} / \varepsilon_{\infty}}=2.1\right)$, whereas the relation holds well for the $\mathrm{A}_{1}{ }^{(4)}$ mode $\left(\omega_{L} / \omega_{T}=\right.$ $1.4, \sqrt{\varepsilon_{0} / \varepsilon_{\infty}}=1.2$ ). 


\subsection{Surface phonon modes on a grating}

Fig. 4 presents reduced band diagrams of the $\mathrm{A}_{1}{ }^{(1)}$ and $\mathrm{A}_{1}{ }^{(4)}$ surface phonons on a grating, which is based on a simple gapless model. To shift most of the dispersion curves (Fig. 3) within the light cone according to Eq. (5), we chose a grating period $\Lambda$ such that the zone edge $\pi / \Lambda$ was located close to $k_{\text {min }}$ (the surface phonon wavevector at $\omega_{T}$ ). In Fig. 4 , the folded dispersion curves extend over the frequency ranges of 7.7-14.6 THz $(\Lambda=19.4 \mu \mathrm{m})$ and $19.1-22.7 \mathrm{THz}(\Lambda=7.8$ $\mu \mathrm{m})$ relevant to the $\mathrm{A}_{1}{ }^{(1)}$ and $\mathrm{A}_{1}{ }^{(4)}$ modes, respectively. Their properties of surface phonon emissions were discussed based on electromagnetic simulations, as shown below. (a)

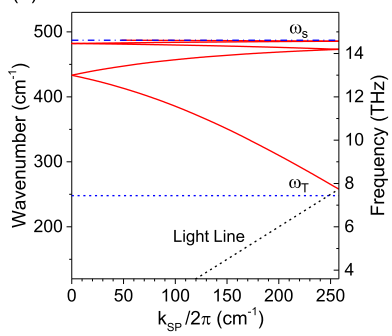

(b)

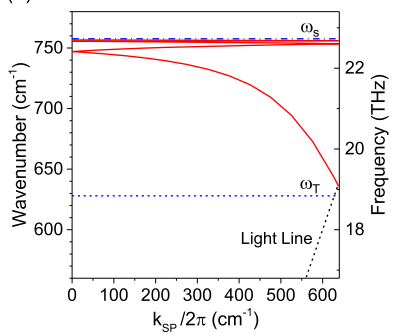

Fig. 4 Dispersion relations of surface phonon modes on a grating. (a) $\mathrm{A}_{1}{ }^{(1)}$ mode, grating period $\Lambda=19.4 \mu \mathrm{m}$. (b) $\mathrm{A}_{1}{ }^{(4)}$ mode, $\Lambda=7.8 \mu \mathrm{m}$.

\section{THz-wave emissions from surface phonons}

We now analyze the THz-wave emissions from surface phonons on a grating. The electromagnetic simulations were conducted using a finite-difference time-domain method (Fujitsu, Poynting for Optics). An LN grating on the $\mathrm{x}$ plane (Fig. 1) was assumed because the polarization of the $\mathrm{A}_{1}$ mode is along the c-axis (i.e., the z-axis). Also supposing an infinite length along the $y$-axis, we performed two-dimensional simulations in the $\mathrm{x}-\mathrm{z}$ plane. To excite surface phonons, we used a point source [33] on the flat surface [orange filled circle in Fig. 5(a)]. The propagating surface waves were diffracted at a structured grating (period $\Lambda=19.4 \mu \mathrm{m}$, groove depth $\mathrm{t}=3 \mu \mathrm{m}$ ), which might yield surface phonon emissions.

The resulting electric field distributions are shown in Figs. 5(b)-5(d). To illustrate both the surface waves and their emissions with TM polarizations, we extracted the amplitude of the $E_{z}$-fields, although the radiative fields had both the $\mathrm{E}_{\mathrm{X}}-$ and $\mathrm{E}_{\mathrm{z}}$-components. As expected, the emissions were largely frequency dependent. Below the TO phonon frequency $(7.4 \mathrm{THz})$, the emission as well as the surface wave were suppressed [Fig. 5(b)]; however, the emission was evident at 9.5 THz [Fig. 5(c)]. Surprisingly, THz-wave emission was also found at $19 \mathrm{THz}$ [Fig. 5(d)], despite the grating period having been designed for the $\mathrm{A}_{1}{ }^{(1)}$ mode. These emission frequencies were both in the negative-permittivity regions relevant to the $A_{1}{ }^{(1)}$ and $A_{1}{ }^{(4)}$ modes. Note that the radiation angles were different (i.e., dispersive), similar to those in Ref. [28].

To confirm the THz-wave emissions arising from the $\mathrm{A}_{1}$ surface phonons, we analyzed the frequencies and wavevectors of the $\mathrm{E}_{\mathrm{z}}$-fields, monitored at a detection area located in the air [green line in Fig. 5(a)]. Fourier analyses of
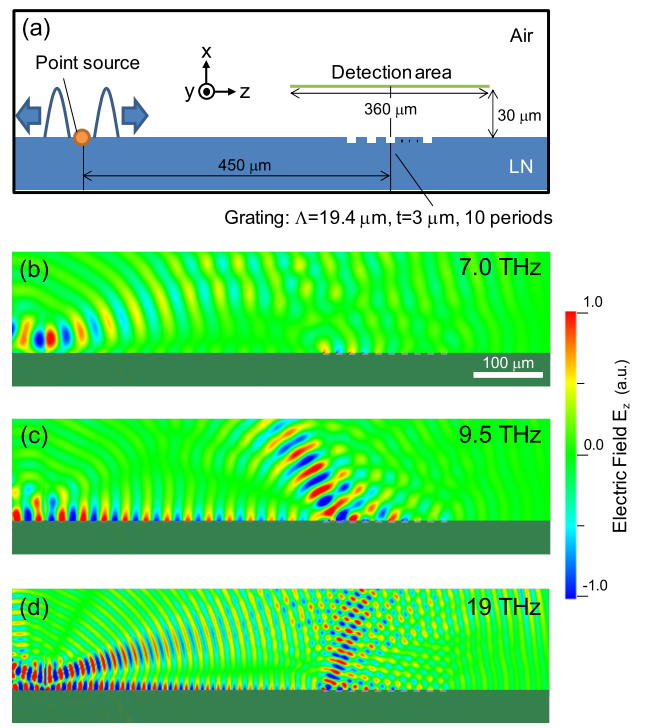

Fig. 5 (a) Schematic of the studied structure. (b) $E_{z}$ distribution at 7.0 $\mathrm{THz}$, (c) $9.5 \mathrm{THz}$, (d) $19 \mathrm{THz}$. The grating period $\Lambda=19.4 \mu \mathrm{m}$.

the temporal $\mathrm{E}_{\mathrm{z}}$-fields, normalized by the incident fields at a flat portion, yielded the emission spectra. To eliminate contributions from the surface waves as well as the direct radiations from the point source [see Figs. 5(b)-5(d)], we subtracted the spectrum for the flat surface from that for the grating surface, as shown in Fig. 6. In the 7.4-12.0 THz range, radiation relevant to the $\mathrm{A}_{1}{ }^{(1)}$ mode was prominent. Furthermore, the second peak at approximately $19 \mathrm{THz}$, attributed to the $\mathrm{A}_{1}{ }^{(4)}$ mode, was also noteworthy. The negative portions $(<7.3 \mathrm{THz},>20.6 \mathrm{THz})$ might have resulted from material absorption or reflection at the grating that was under investigation.

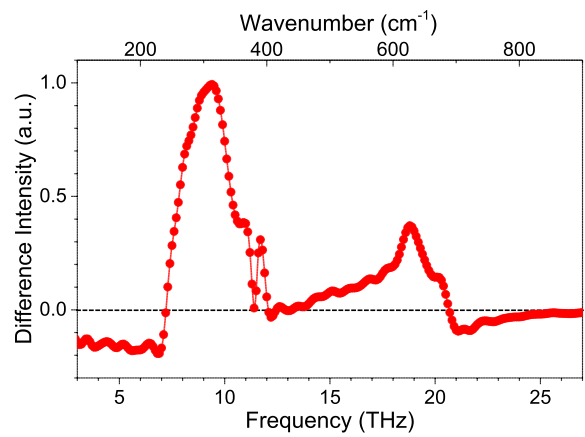

Fig. 6 THz-wave spectrum emitted from the $A_{1}$ surface phonons on a grating $(\Lambda=19.4 \mu \mathrm{m})$.

Furthermore, the spatial Fourier transform of the $\mathrm{E}_{\mathrm{z}}$-fields ( $\mathrm{k}_{\mathrm{z}}$-components) at each frequency led to the dispersion relations, as shown in Fig. 7. The bright portion at approximately $9 \mathrm{THz}$ deviated from the light line and was in excellent agreement with the $\mathrm{A}_{1}{ }^{(1)}$ surface phonon branch, shifted by the first-order diffraction according to Eq. (5). Despite the limited frequency range, the second bright portion at approximately $19 \mathrm{THz}\left(k_{S P} / 2 \pi \sim 120 \mathrm{~cm}^{-1}\right)$ also agreed well with the first-order diffracted surface phonon branch of the $\mathrm{A}_{1}{ }^{(4)}$ mode. These behaviors were similar to the plasmonic resonance on a metal grating $[34,35]$. Therefore, we decided that the present $\mathrm{THz}$-wave radiations were those supported 


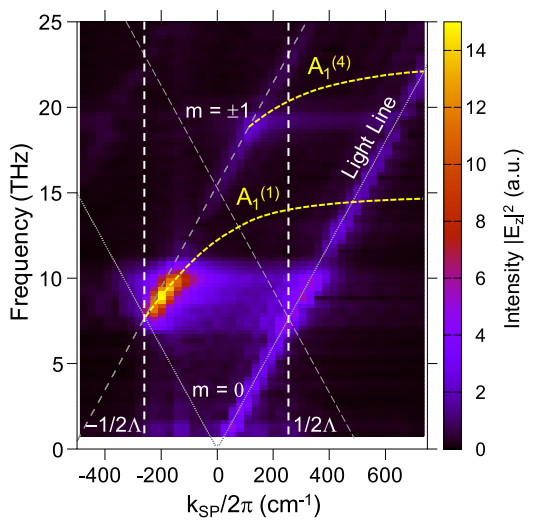

Fig. 7 Dispersion relations of the $A_{1}$ surface-phonon emissions from a structured grating $(\Lambda=19.4 \mu \mathrm{m})$, depicted in the vicinity of the first Brillouin zone.

by the $A_{1}$ surface phonons in LN. A single periodicity of an LN grating opens the emission channels at approximately 9 and $19 \mathrm{THz}$. We did not find significant emissions from the $A_{1}{ }^{(4)}$ surface phonons on an LN grating of $\Lambda=7.8 \mu \mathrm{m}$. However, we consider that emissions might be possible if the structural parameters (especially, the groove depths) were optimized. Although we have herein studied the linear optical phenomena on $\mathrm{THz}$ electromagnetic waves, the results of our research might also hold for nonlinear-optic $\mathrm{THz}$-wave generation $[1,2$, 3, 4, 5, 6, 7, 8, 9]. Laser-induced nonlinear polarizations are equivalent to the coherent excitation sources exploited here. Therefore, surface phonon emission might present a potential path to novel $\mathrm{THz}$-wave sources at the forbidden frequencies in solids, including nonlinear optical crystals and semiconductors.

\section{Conclusion}

We have studied THz-wave generation in the forbidden bands of LN crystals. It has been shown that the surface phonons can be excited in relation to the lowest and highest $\mathrm{A}_{1}$ modes, which can radiate widely tunable $\mathrm{THz}$ waves with a grating of single periodicity. These findings provide a basis for realizing coherent $\mathrm{THz}$-wave sources at unexplored forbidden frequencies.

\section{Acknowledgments}

This work is partly supported by JSPS KAKENHI Grant Numbers JP19K05286 and JP18K04967 as well as the cooperative research aid from RIKEN.

\section{References}

[1] K. Kawase, et al.: "Coherent tunable THz-wave generation from $\mathrm{LiNbO}_{3}$ with monolithic grating coupler," Appl. Phys. Lett. 68 (1996) 2483 (DOI: 10.1063/1.115828).

[2] J. Shikata, et al.: "Tunable terahertz-wave parametric oscillators using $\mathrm{LiNbO}_{3}$ and $\mathrm{MgO}: \mathrm{LiNbO}_{3}$ crystals," IEEE Trans. Microw. Theory Techn. 48 (2000) 653 (DOI: 10.1109/22.841956).

[3] K. Kawase, et al.: "Terahertz wave parametric source," J. Phys. D 35 (2002) R1 (DOI: 10.1088/0022-3727/35/3/201).

[4] S. Hayashi, et al.: "Ultrabright continuously tunable terahertz-wave generation at room temperature," Sci. Rep. 4 (2014) 5045 (DOI: 10.1038/srep05045).
[5] Y. Takida, et al.: "Terahertz-wave parametric gain of stimulated polariton scattering," Phys. Rev. A 93 (2016) 043836 (DOI: 10.1103/ physreva.93.043836).

[6] K. Nawata, et al.: "Tunable backward terahertz-wave parametric oscillation,” Sci. Rep. 9 (2019) 726 (DOI: 10.1038/s41598-018-370687).

[7] T. Tanabe, et al.: "Frequency-tunable terahertz wave generation via excitation of phonon-polaritons in GaP,” J. Phys. D 36 (2003) 953 (DOI: 10.1088/0022-3727/36/8/302).

[8] T. Taniuchi, et al.: "Continuously tunable $\mathrm{THz}$ and far-infrared wave generation from DAST crystal," Electron. Lett. 40 (2004) 60 (DOI: 10.1049/el:20040380).

[9] K. Miyamoto, et al.: "Widely tunable terahertz-wave generation using an N-benzyl-2-methyl-4-nitroaniline crystal," Opt. Lett. 33 (2008) 252 (DOI: 10.1364/ol.33.000252).

[10] E.B. Brown, et al.: "Photomixing up to $3.8 \mathrm{THz}$ in low temperaturegrown GaAs," Appl. Phys. Lett. 66 (1995) 285 (DOI: 10.1063/ 1.113519).

[11] K.A. McIntosh, et al.: "Terahertz measurements of resonant planar antennas coupled to low-temperature-grown GaAs photomixers," Appl. Phys. Lett. 69 (1996) 3632 (DOI: 10.1063/1.117006).

[12] I.S. Gregory, et al.: "Continuous-wave terahertz system with a 60 dB dynamic range," Appl. Phys. Lett. 86 (2005) 204104 (DOI: 10.1063/1.1935032).

[13] R. Köhler, et al.: "Terahertz semiconductor-heterostructure Laser," Nature 417 (2002) 156 (DOI: 10.1038/417156a).

[14] S. Kumar: "Recent progress in terahertz quantum cascade Lasers," IEEE J. Sel. Topics Quantum Electron. 17 (2011) 38 (DOI: 10.1109/ jstqe.2010.2049735)

[15] T.-T. Lin and H. Hirayama: "Improvement of operation temperature in GaAs/AlGaAs THz-QCLs by utilizing high Al composition barrier," Phys. Status Solidi C 10 (2013) 1430 (DOI: 10.1002/pssc. 201300216).

[16] J. Nishizawa, et al.: "GaAs TUNNETT diodes oscillating at 430-655 $\mathrm{GHz}$ in CW fundamental mode," IEEE Microw. Wireless Compon. Lett. 15 (2005) 597 (DOI: 10.1109/lmwc.2005.855381).

[17] J. Nishizawa, et al:: "706-GHz GaAs CW fundamental-mode TUNNETT diodes fabricated with molecular layer epitaxy," Phys. Status Solidi C 5 (2008) 2802 (DOI: 10.1002/pssc.200779256).

[18] T. Ishibashi, et al.: "High-speed response of uni-traveling-carrier photodiodes,” Jpn. J. Appl. Phys. 36 (1997) 6263 (DOI: 10.1143/jjap.36. 6263).

[19] T. Ishibashi, et al.: "Unitraveling-carrier photodiodes for terahertz applications," IEEE J. Sel. Topics Quantum Electron. 20 (2014) 3804210 (DOI: 10.1109/jstqe.2014.2336537).

[20] T. Kurokawa, et al:: "Over $300 \mathrm{GHz}$ bandwidth UTC-PD module with $600 \mathrm{GHz}$ band rectangular waveguide output," Electron. Lett. 54 (2018) 705 (DOI: 10.1049/el.2018.0932).

[21] M. Asada, et al.: "Resonant tunneling diodes for sub-terahertz and terahertz oscillators,” Jpn. J. Appl. Phys. 47 (2008) 4375 (DOI: 10.1143/jjap.47.4375).

[22] S. Suzuki, et al.: "Fundamental oscillation of resonant tunneling diodes above $1 \mathrm{THz}$ at room temperature," Appl. Phys. Lett. 97 (2010) 242102 (DOI: 10.1063/1.3525834).

[23] R. Izumi, et al.: "Resonant-tunneling-diode terahertz oscillator with a cylindrical cavity for high-frequency oscillation," AIP Adv. 9 (2019) 085020 (DOI: 10.1063/1.5114963).

[24] Y.M. Meziani, et al.: "Room temperature generation of terahertz radiation from a grating-bicoupled plasmon-resonant emitter: Size effect," Appl. Phys. Lett. 90 (2007) 061105 (DOI: 10.1063/1.2459879).

[25] A.A. Dubinov, et al.: "Terahertz plasmon-emitting graphene-channel transistor," Opto-Electron. Rev. 27 (2019) 345 (DOI: 10.1016/ j.opelre.2019.11.003)

[26] C. Kittel: Introduction to Solid State Physics (Wiley, New York, 2005) 8th ed. 410.

[27] Y.R. Shen: The Principles of Nonlinear Optics (Wiley, New York, 1984) 481.

[28] J.-J. Greffet, et al.: "Coherent emission of light by thermal sources," Nature 416 (2002) 61 (DOI: 10.1038/416061a).

[29] R. Carminati and J.-J. Greffet: "Near-field effects in spatial coherence of thermal sources," Phys. Rev. Lett. 82 (1999) 1660 (DOI: 
10.1103/physrevlett.82.1660).

[30] A.S. Baker, Jr. and R. Loudon: "Dielectric properties and optical phonons in $\mathrm{LiNbO}_{3}$," Phys. Rev. 158 (1967) 433 (DOI: 10.1103/ physrev.158.433).

[31] J. Le Gall, et al.: "Experimental and theoretical study of reflection and coherent thermal emission by a $\mathrm{SiC}$ grating supporting a surface-phonon polartion," Phys. Rev. B 55 (1997) 10105 (DOI: 10.1103/physrevb.55.10105).

[32] Y. Ichikawa, et al.: "Direct observation of the soft-mode dispersion in the incipient ferroelectric $\mathrm{KTaO}_{3}$," Phys. Rev. B 71 (2005) 092196 (DOI: 10.1103/physrevb.71.092106).

[33] B. Hecht, et al.: "Local excitation, scattering, and interference of surface plasmons," Phys. Rev. Lett. 77 (1996) 1889 (DOI: 10.1103/ physrevlett.77.1889).

[34] S.A. Maier: "Plasmonics: metal nanostructures for sub-wavelength photonic devices," IEEE J. Sel. Topics Quantum Electron. 12 (2006) 1214 (DOI: 10.1109/jstqe.2006.879582).

[35] S.A. Maier: Plasmonics: Fundamentals and Applications (Springer Verlag, 2007) 30. 